\title{
Hetero Diels-Alder Reactions of Nitrosoamidines: A Efficient Method for the Synthesis of Functionalized Guanidines
}

\author{
Craig A. Miller and Robert A. Batey
}

\section{Experimental Procedure}

\section{General Synthetic Methods}

The following general experimental procedure applies for all experiments described in this paper. Unless otherwise stated, all reactions were performed under nitrogen using flame-dried glassware. Reaction solvents were distilled under an inert atmosphere before use and transferred via syringe using standard techniques unless otherwise stated. THF, diethyl ether, and benzene were distilled from sodiumbenzophenone ketyl under argon. $\mathrm{CH}_{2} \mathrm{Cl}_{2}$ and $\mathrm{MeCN}$ were distilled from $\mathrm{CaH}_{2}$ under argon and $\mathrm{MeOH}$ and $\mathrm{EtOH}$ were dried over $4 \AA$ molecular seizes and stored under nitrogen. All other solvents were used as obtained except hexanes, which was distilled prior to use. All reagents, unless otherwise stated, were used as received (Aldrich, Fisher Scientific Ltd. or Lancaster). Crystalline $\mathrm{Pr}_{4} \mathrm{NIO}_{4}$ was conveniently prepared by treatment of equimolar amounts of $\mathrm{Pr}_{4} \mathrm{NOH}$ and $\mathrm{HIO}_{4}$ in water (Keck, G. E.; Fleming, S. A. Tetrahedron Lett. 1978, 19, 4763-4766).

IR spectra were obtained in a Perkin-Elmer Spectrum 1000, with samples loaded as films on $\mathrm{NaCl}$ plates. ${ }^{1} \mathrm{H}$ and ${ }^{13} \mathrm{C}$ NMR spectra were obtained using a Varian Mercury 300 or Unity 400 or 500 spectrometers as solutions in $\mathrm{CDCl}_{3}$. Chemical shifts are expressed in ppm values downfield from TMS. Spectra are referenced to 7.26 for $\mathrm{CDCl}_{3}$ for proton chemical shifts, and $77.00 \mathrm{ppm}$ for $\mathrm{CDCl}_{3}$ for carbon chemical shifts. Peak multiplicities are designated by the following abbreviations: $s$, singlet; $d$, doublet; $t$, triplet; q, quartet; m, multiplet; dd, doublet of doublets; dt, doublet of triplets; b, broad (this abbreviation is also used for designation of IR peaks); r, rotamer, $J$, coupling constant in Hz. Line broadening is apparent in some NMR spectra and is due to rotamers around the carbamate nitrogen. Low resolution mass spectra (MS) were recorded on a 
Bell or Howell 21-490 spectrometer, high resolution mass spectra (HRMS) were recorded using a AEI MS3074 spectrometer. Melting points were obtained on a Fisher-Johns melting point apparatus and are uncorrected.

Flash column chromatography on silica gel (60 $\AA, 230-400$ mesh, obtained from Whatman Company Silicycle) was preformed with distilled hexanes, or reagent grade solvents. Analytical thin-layer chromatography (TLC) was performed on pre-coated aluminum-backed silica gel plates (Alugram SIL G/UV ${ }_{254}$ purchased from Rose Scientific Limited), visualized with with a UV lamp (254 nm), iodine, ninhydrin, potassium permanganate, phosphomolybic acid (Aldrich), or vanillin. References following the compound names indicate literature articles where ${ }^{1} \mathrm{H}$ and ${ }^{13} \mathrm{C}$ NMR data have previously been reported.

\section{General Synthetic Preparations}

\section{$N, N$ '-di-tert-butoxycarbony-S-methylisothiourea (3).}

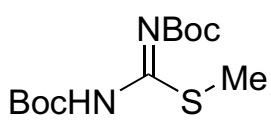

To a solution of $S$-methylisothiourea $(2.00 \mathrm{~g}, 7.20 \mathrm{mmol})$ in $\mathrm{CH}_{2} \mathrm{Cl}_{2}(40 \mathrm{~mL})$ and saturated $\mathrm{NaHCO}_{3}(40 \mathrm{~mL})$ was added $(\mathrm{Boc})_{2} \mathrm{O}(9.91 \mathrm{~mL}, 43.0 \mathrm{mmol})$ and the solution stirred for 12 hours. The solution was then diluted with $\mathrm{H}_{2} \mathrm{O}(40 \mathrm{~mL})$ and extracted with $\mathrm{CH}_{2} \mathrm{Cl}_{2}(3 \times 30 \mathrm{~mL})$. The combined organic extracts were washed with brine and dried with $\mathrm{MgSO}_{4}$. The solvent was removed under vacuum and the crude material was purified by silica gel chromatography to give as a white solid $(2.58 \mathrm{~g}, 61 \%) .{ }^{1} \mathrm{H}$ NMR $\left(300 \mathrm{MHz}, \mathrm{CDCl}_{3}\right) \delta 11.51(1 \mathrm{H}, \mathrm{bs}), 2.39(3 \mathrm{H}, \mathrm{s}), 1.52(9 \mathrm{H}, \mathrm{s}), 1.50(9 \mathrm{H}, \mathrm{s}) ;{ }^{13} \mathrm{C} \mathrm{NMR}$ $\left(75 \mathrm{MHz}, \mathrm{CDCl}_{3}\right) \delta 171.38,150.32,146.79,85.43,83.50,28.52,27.91,14.93$. Compound has previously characterized by: Bergon, R.J.; McManis, J.S. J. Org. Chem. 1987, 52, 1700-1703. 


\section{$N, N$ '-di-tert- butoxycabonyl- $N$ '’-hydroxylguandine (4).}

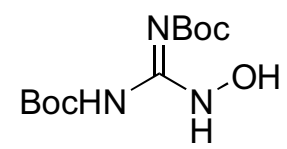

To a solution of $N$-N'-bis-Boc-S-methylisothiourea $(2.00 \mathrm{~g}, 6.80 \mathrm{mmol})$ in $30 \mathrm{ml}$ of dry MeCN was added $\mathrm{HgCl}_{2}(1.38 \mathrm{~g}, 20.0 \mathrm{mmol}), \mathrm{NH}_{2} \mathrm{OH} \mathrm{HCl}(1.40 \mathrm{~g}, 20.4 \mathrm{mmol})$ and Amberlyst A21 ion exchange resin $(7 \mathrm{~g})$. The reaction was allowed to stir at room temperature overnight and then filtered through a pad of celite. The solution was then diluted with $\mathrm{H}_{2} \mathrm{O}(50 \mathrm{~mL})$ and extracted with EtOAc $(3 \times 30 \mathrm{ml})$. The combined organics were washed with brine, dried with $\mathrm{MgSO}_{4}$ and the solvent removed under vacuum. The resulting white solid was purified by silica gel chromatography to give the desired compound as a white powder $(1.31 \mathrm{~g}, 61 \%) . \mathrm{mp}=175{ }^{\circ} \mathrm{C} ; \mathrm{R}_{f}=0.41$ (EtOAc:hexanes; 1:1); IR (Thin Film) v 3229, 2979, 1718, 1684, 1540, 1534, 1245, $1148 \mathrm{~cm}^{-1} ;{ }^{1} \mathrm{H}$ NMR $\left(300 \mathrm{MHz}, \mathrm{CDCl}_{3}\right) \delta 9.87(1 \mathrm{H}, \mathrm{bs}), 9.10(1 \mathrm{H}, \mathrm{s}), 7.95(1 \mathrm{H}, \mathrm{bs}), 1.49(9 \mathrm{H}, \mathrm{s}), 1.47(9 \mathrm{H}, \mathrm{s})$; ${ }^{13} \mathrm{C}$ NMR $\left(75 \mathrm{MHz}, \mathrm{CDCl}_{3}\right) \delta 152.30,150.07,140.45,83.32,81.62,28.64,28.54$; MS (EI) m/e (rel intensity) 275 (3), 219 (13), 163 (21), 146 (30), 119 (49), 101 (42), 57 (100); HRMS (EI) $m / e$ calcd $\left(\mathbf{M}^{+}\right) 276.1559$, found 276.1558.

\section{General Procedure for Cycloaddition of 4.}

A solution of $\mathrm{Pr}_{4} \mathrm{NIO}_{4}(0.15 \mathrm{~g}, 0.40 \mathrm{mmol})$ and diene $(0.40 \mathrm{mmol})$ in $\mathrm{MeOH}(5 \mathrm{~mL})$ was cooled to $0^{\circ} \mathrm{C}$ in an ice water bath. To this was added $N, N$ ' di-Boc- $N$ ''-hydroxylguandine $4(0.100 \mathrm{~g}, 0.36 \mathrm{mmol})$ in small portions over 2 hours and the reaction was allowed to warm to room temperature overnight. The solution was quenched with $\mathrm{H}_{2} \mathrm{O}(20 \mathrm{~mL})$ and extracted with $\mathrm{CH}_{2} \mathrm{Cl}_{2}(3 \times 20 \mathrm{~mL})$ and the combined organic layers were washed with saturated $\mathrm{Na}_{2} \mathrm{~S}_{2} \mathrm{O}_{7}$, brine and dried with $\mathrm{MgSO}_{4}$. The solvent was removed under vacuum and the resulting product was purified by silica gel chromatography. 
[tert-Butoxycarbonylimino-(2-oxa-aza-bicyclo[2.2.2]oct-5-en-3-yl)-methyl]-carbamic acid tert-butyl ester (6).

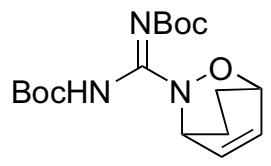

Obtained as a white solid in $85 \%$ yield: $\mathrm{Mp}=124{ }^{\circ} \mathrm{C}$; $\mathrm{R}_{f}=0.34$ (EtOAc:hexanes; 1:1); IR (Thin Film) v 3398, 2976, 2962, 1760, 1633, 1497, 1367, 1283, 1054, $935 \mathrm{~cm}^{-1}$; ${ }^{1} \mathrm{H}$ NMR $\left(300 \mathrm{MHz}, \mathrm{CDCl}_{3}\right) \delta 8.01(1 \mathrm{H}, \mathrm{s}), 6.65(1 \mathrm{H}, \mathrm{ddd}, J=8.0,5.9,1.6 \mathrm{~Hz}), 6.50(1 \mathrm{H}, \mathrm{ddd}, J=$ 7.5, 5.6, 1.6 Hz), 5.24 (2H, d , $J=4.0 \mathrm{~Hz}$, broad), 4.79 (2H, m), 2.23-2.15 (4H, m), 1.47 $(18 \mathrm{H}, \mathrm{s}) ;{ }^{13} \mathrm{C} \mathrm{NMR}\left(75 \mathrm{MHz}, \mathrm{CDCl}_{3}\right) \delta 151.81,133.20,131.27,72.62,50.56,28.61$, 24.00, 20.68 (2 equivalent carbons not observed); MS (EI) m/e (rel intensity) 353 (61), 298 (37), 280 (87), 253 (46), 241 (80), 197 (60), 179 (24), 79 (46), 57 (100); HRMS (EI) m/e calcd $\left(\mathrm{M}^{+}\right)$353.1951, found 353.1962.

[tert-Butoxycarbonylimino-(2-oxa-3-aza-bicyclo[2.2.1]hept-5-en-3-yl)-methyl]carbamic acid tert-butyl ester (Table 1, entry 2).

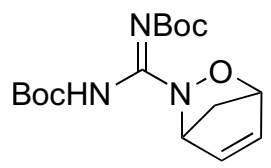

Obtanied as a light yellow solid in $81 \%$ yield: $\mathrm{Mp}=111^{\circ} \mathrm{C} ; \mathrm{R}_{f}=0.30$ (EtOAc:hexanes; 1:2); IR (Thin Film) v 3398, 2976, 1760, 1683, 1497, 1367, 1283, $1149 \mathrm{~cm}^{-1}$; ${ }^{1} \mathrm{H}$ NMR $\left(300 \mathrm{MHz}, \mathrm{CDCl}_{3}\right) \delta 8.35(1 \mathrm{H}, \mathrm{bs}), 6.60(1 \mathrm{H}, \mathrm{ddd}, J=7.3,4.0,1.8 \mathrm{~Hz}), 6.42(1 \mathrm{H}, \mathrm{ddd}, J$ $=5.6,2.3,1.5 \mathrm{~Hz}), 5.33(1 \mathrm{H}, \mathrm{m}), 5.28(1 \mathrm{H}, \mathrm{m}), 2.10(1 \mathrm{H}, \mathrm{ddd}, J=8.8,3.8,1.9 \mathrm{~Hz}), 1.83$ $(1 \mathrm{H}, \mathrm{bd}, J=8.8 \mathrm{~Hz}), 1.48(18 \mathrm{H}, \mathrm{s}) ;{ }^{13} \mathrm{C} \mathrm{NMR}\left(75 \mathrm{MHz}, \mathrm{CDCl}_{3}\right) \delta 153.64,136.58$, 133.23, 84.81, 66.11, 48.50, 28.08, 27.98 (1 quaternary carbon not observed); MS (EI) 
m/e (rel intensity) 266 (7), 205 (9), 149 (34), 131 (13), 105 (21), 70 (19), 57 (100); HRMS (EI) $m / e$ calcd $\left(\mathrm{M}^{+}\right)$339.1794, found 339.1799.

tert-Butoxycarbonylimino-(4,5-dimethyl-3,6-dihydro-[1,2]oxazin-2-yl)-methyl]carbamic acid tert-butyl ester (Table 1, entry 3).

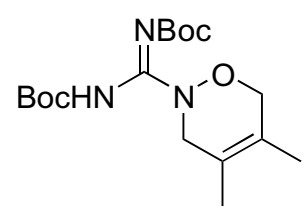

Obtained as a white solid in $78 \%$ yield: $\mathrm{mp}=178^{\circ} \mathrm{C} ; \mathrm{R}_{f}=0.29$ (EtOAc:hexanes; 1:2); IR (Thin Film) v 3281, 2972, 2931, 1756, 1599, 1368, 1141, 1046, $776 \mathrm{~cm}^{-1} ;{ }^{1} \mathrm{H}$ NMR (300 $\left.\mathrm{MHz}, \mathrm{CDCl}_{3}\right) \delta 9.05(1 \mathrm{H}, \mathrm{bs}), 4.31(2 \mathrm{H}, \mathrm{s}), 3.98(2 \mathrm{H}, \mathrm{bs}), 1.66(3 \mathrm{H}, \mathrm{s}), 1.59(3 \mathrm{H}, \mathrm{s}), 1.50$ $(18 \mathrm{H}, \mathrm{s}) ;{ }^{13} \mathrm{C}$ NMR $\left(75 \mathrm{MHz}, \mathrm{CDCl}_{3}\right) \delta 162.58,152.72,122.91,121.36,73.05,49.18$, 28.58, 15.92, 14.18; MS (EI) m/e (rel intensity) 356 (4), 300 (6), 244 (9), 226 (29), 181 (15), 113 (31), 96 (47), 57 (100); HRMS (EI) $\mathrm{m} / \mathrm{e}$ calcd (M+) 356.2185, found 356.2190.

tert-Butoxycarbonylimino-(3,6-dimethyl-3,6-dihydro-[1,2]oxazin-2-yl)-methyl]carbamic acid tert-butyl ester (Table 1, entry 4).

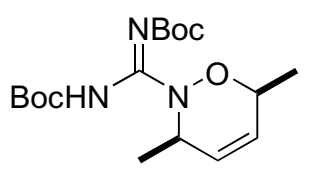

Isolated as a white solid in $78 \%$ yield. $m p=191{ }^{\circ} \mathrm{C} ; \mathrm{R}_{f}=0.41$ (EtOAc:hexanes; 2:3); IR (Thin Film) v 3210, 2923, 2871, 1721, 1653, 1321, 1109, 985, $880 \mathrm{~cm}^{-1} ;{ }^{1} \mathrm{H}$ NMR (300 $\left.\mathrm{MHz}, \mathrm{CDCl}_{3}\right) \delta 9.05(1 \mathrm{H}, \mathrm{s}), 5.77(1 \mathrm{H}, \mathrm{m}), 5.65(1 \mathrm{H}, \mathrm{d}, J=10.3 \mathrm{~Hz}), 4.82(2 \mathrm{H}, \mathrm{m}), 1.50$ $(18 \mathrm{H}, \mathrm{s}), 1.32(3 \mathrm{H}, \mathrm{d}, J=6.4 \mathrm{~Hz}), 1.24(3 \mathrm{H}, \mathrm{d}, J=6.6 \mathrm{~Hz}) ;{ }^{13} \mathrm{C} \mathrm{NMR}\left(75 \mathrm{MHz}, \mathrm{CDCl}_{3}\right)$ $\delta 161.27,151.67,149.95,81.91,79.43,75.41,49.89,28.21,28.02,18.61,17.10 ;$ MS (EI) 
m/e (rel intensity) 355 (1), 226 (21), 208 (28), 181 (29), 155 (9), 138 (11), 113 (16), 98 (21), 82 (36), 57 (100); HRMS (EI) m/e calcd ( $\left.\mathrm{M}^{+}\right)$355.2107, found 355.2109.

tert-Butoxycarbonylimino-(6-ethyl-3,6-dihydro-[1,2]oxazin-2-yl)-methyl]-carbamic acid tert-butyl ester (Table 2, entry 1).

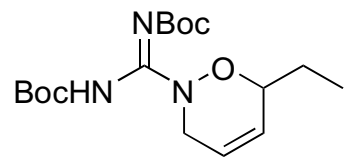

Obtained as a white solid in $75 \%$ yield: $\mathrm{mp}=183{ }^{\circ} \mathrm{C} ; \mathrm{R}_{f}=0.57$ (EtOAc:hexanes; 1:2); IR (Thin Film) v 3227, 2997, 2934, 1757, 1601, 1289, $1148 \mathrm{~cm}^{-1} ;{ }^{1} \mathrm{H}$ NMR (300 MHz, $\left.\mathrm{CDCl}_{3}\right) \delta 8.55(1 \mathrm{H}, \mathrm{s}), 5.84-5.76(1 \mathrm{H}, \mathrm{m}), 4.68(1 \mathrm{H}, \mathrm{bs}), 4.37-4.33(1 \mathrm{H}, \mathrm{m}), 3.98-3.94$ $(1 \mathrm{H}, \mathrm{m}), 1.63-1.57(2 \mathrm{H}, \mathrm{m}), 1.50(18 \mathrm{H}, \mathrm{s}), 0.97(3 \mathrm{H}, \mathrm{t}, J=4.5 \mathrm{~Hz}) ;{ }^{13} \mathrm{C}$ NMR $(100 \mathrm{MHz}$, $\left.\mathrm{CDCl}_{3}\right) \delta 153.13,127.69,121.90,80.36,45.61,38.41,26.37,9.60$ (1 quaternary carbon not observed); MS (EI) m/e (rel intensity) 358 (7), 300 (9), 226 (27), 126 (34), 81 (39), 57 (100); HRMS (EI) m/e calcd ( $\left.\mathrm{M}^{+}\right) 356.2185$, found 356.2198 .

[tert-Butoxycarbonylimino-(6-tert-butyl-3,6-dihydro-[1,2]oxazin-2-yl)-methyl]carbamic acid tert-butyl ester (Table 2, entry 2).

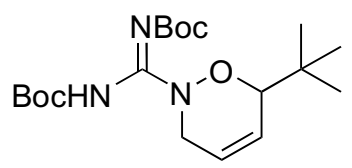

Obtained as a clear oil in 79\% yield: $\mathrm{R}_{f}=0.33$ (Hexanes:EtOAc; 3:1); IR (Thin Film) v 3232.3, 2972, 1756, 1641, 1604, 1291, 1150, $983 \mathrm{~cm}^{-1} ;{ }^{1} \mathrm{H}$ NMR (300 MHz, $\mathrm{CDCl}_{3}$ ) $\delta 9.08(1 \mathrm{H}, \mathrm{s}), 5.91-5.84(2 \mathrm{H}, \mathrm{m}), 4.48-4.47(1 \mathrm{H}, \mathrm{m}), 4.43-4.39(1 \mathrm{H}, \mathrm{m}), 3.90-3.84(1 \mathrm{H}$, m), $1.49(18 \mathrm{H}, \mathrm{s}), 0.95(9 \mathrm{H}, \mathrm{s}) ;{ }^{13} \mathrm{C} \mathrm{NMR}\left(100 \mathrm{MHz}, \mathrm{CDCl}_{3}\right) \delta$ 153.07, 125.15, 123.11, 
85.78, 45.09, 33.97, 28.16, 25.45 (1 quaternary carbon not observed); MS (EI) m/e (rel intensity) 384 (4), 272 (5), 254 (17), 227 (15), 124 (18), 109 (25), 108 (12), 59 (16), 57 (100); HRMS (EI) m/e calcd ( $\left.\mathrm{M}^{+}\right) 384.2498$, found 384.2489 .

tert-Butoxycarbonylimino-(6-methoxy-3,6-dihydro-[1,2]oxazin-2-yl-methyl]carbamic acid tert-butyl ester (Table 2, entry 3 ).

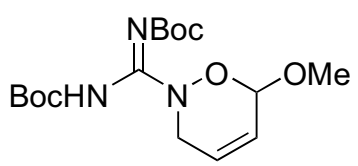

Obtained as a white solid in $86 \%$ yield: $\mathrm{mp}=185^{\circ} \mathrm{C} ; \mathrm{R}_{f}=0.26$ (EtOAc:hexanes; $1: 1$ ); IR (Thin Film) v 3431, 3241, 2978, 2933, 1759, 1602, 1505, 1367, 1287, 1149, $999 \mathrm{~cm}^{-1} ;{ }^{1} \mathrm{H}$ NMR (300 MHz, $\left.\mathrm{CDCl}_{3}\right) \delta 8.64(1 \mathrm{H}, \mathrm{s}), 5.99(1 \mathrm{H}, \mathrm{m}), 5.74(1 \mathrm{H}, \mathrm{ddd}, J=10.1,4.5,2.2$ $\mathrm{Hz}), 5.18(1 \mathrm{H}, \mathrm{s}), 4.51(1 \mathrm{H}$, ddd, $J=19.2,3.6,1.2 \mathrm{~Hz}$, broad), 3.94 (1H, ddd, $J=18.1$, 4.4, $2.2 \mathrm{~Hz}), 3.50(3 \mathrm{H}, \mathrm{s}), 1.45(18 \mathrm{H}, \mathrm{s}) ;{ }^{13} \mathrm{C} \mathrm{NMR}\left(100 \mathrm{MHz}, \mathrm{CDCl}_{3}\right) \delta 160.88,152.38$, $149.91,126.88,123.36,100.95,82.32,79.94,57.39,44.50,28.35,28.25$; MS (EI) $m / e$ (rel intensity) 358 (3), 257 (8), 228 (34), 201 (90), 157 (33), 84 (43), 69 (32), 57 (100); HRMS (EI) m/e calcd (M $\left.{ }^{+}\right) 358.1978$, found 358.1981.

tert-Butoxycarbonylimino-(4,6-dimethyl-3,6-dihydro-[1,2]oxazin-2-yl)-methyl]carbamic acid tert-butyl ester (Table 3, entry 4).

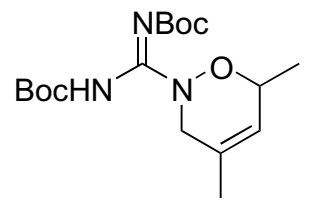

Obtained as a white solid in $70 \%$ yield: $m p=191{ }^{\circ} \mathrm{C} ; \mathrm{R}_{f}=0.20$ (EtOAc:hexanes; 1:2); IR $\left(\right.$ Thin Film) v 3230, 2978, 2932, 1757, 1599, 1508, 1367, 1284, $1148 \mathrm{~cm}^{-1} ;{ }^{1} \mathrm{H}$ NMR (300 
$\left.\mathrm{MHz}, \mathrm{CDCl}_{3}\right) \delta 9.03(1 \mathrm{H}, \mathrm{s}), 5.41(1 \mathrm{H}, \mathrm{ddd}, J=2.9,1.4,1.4 \mathrm{~Hz}), 4.77(1 \mathrm{H}, \mathrm{m}), 4.19(1 \mathrm{H}$, d , $J=17.1 \mathrm{~Hz}$, broad), $3.82(1 \mathrm{H}, \mathrm{d}, J=16.8 \mathrm{~Hz}$, broad), $1.73(3 \mathrm{H}, \mathrm{s}), 1.50(18 \mathrm{H}, \mathrm{s}), 2.42$ $(3 \mathrm{H}, \mathrm{d}, J=6.7 \mathrm{~Hz}) ;{ }^{13} \mathrm{C} \mathrm{NMR}\left(100 \mathrm{MHz}, \mathrm{CDCl}_{3}\right) \delta 152.70,129.42,123.11,75.34,48.83$, 28.56, 20.13, 19.29 (1 quaternary carbon not observed); MS (EI) m/e (rel intensity) 357 (3), 226 (17), 199 (28), 145 (17), 126 (27), 81 (20), 57 (100); HRMS (EI) m/e calcd (M ${ }^{+}$) 356.2171, found 356.2173.

tert-Butoxycarbonylimino-(4-methyl-3,6-dihydro-[1,2]oxazin-2-yl)-methyl]-carbamic acid tert-butyl ester (Table 2, entry 5).

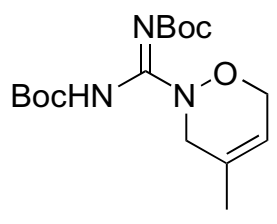

Obtained as a white solid in 71\% yield as a 85:15 mixture of inseparable regioisomers: $\mathrm{mp}=171{ }^{\circ} \mathrm{C} ; \mathrm{R}_{f}=0.21$ (EtOAc:hexanes; 2:3); IR (Thin Film) v 3283, 2978, 2932, 1756, 1600, 1288, 1150, 916, $732 \mathrm{~cm}^{-1} ;{ }^{1} \mathrm{H}$ NMR (300 MHz, $\left.\mathrm{CDCl}_{3}\right) \delta 5.52(1 \mathrm{H}, \mathrm{ddd}, J=4.3$, 2.7, $1.4 \mathrm{~Hz}$, broad), 4.48 (2H, dd , $J=4.5,2.3 \mathrm{~Hz}), 4.02(2 \mathrm{H}, \mathrm{bs}), 1.74$ (3H, bs), 1.50 $(18 \mathrm{H}, \mathrm{s}) ;{ }^{13} \mathrm{C} \mathrm{NMR}\left(75 \mathrm{MHz}, \mathrm{CDCl}_{3}\right) \delta 152.51,129.67,117.75,69.94,49.36,28.54$, 20.27 (3 quaternary carbon not observed); MS (EI) m/e (rel intensity) 342 (4), 286 (5), 229 (17), 212 (85), 194 (67), 185 (38), 167 (56), 99 (100), 82 (27), 57 (56); HRMS (EI) m/e calcd $\left(\mathrm{M}^{+}\right) 342.2029$, found 342.2025. 


\section{3,5-hexadien-1-ol}

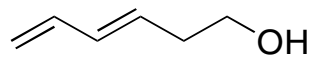

To a solution of diisopropylamine $(12.4 \mathrm{~mL}, 90.0 \mathrm{mmol})$ in dry THF $(60 \mathrm{~mL})$ was added a solution of $n$-butyllithium in hexane $(37 \mathrm{~mL}, 90 \mathrm{mmol})$ at $-10{ }^{\circ} \mathrm{C}$. After the solution was stirred for $30 \mathrm{~min}$, the reaction mixture was cooled to $-78{ }^{\circ} \mathrm{C}$. To this was added HMPA $(20 \mathrm{~mL})$ and stirring was continued for $20 \mathrm{~min}$. Ethyl sorbate $(7 \mathrm{~g}, 50 \mathrm{mmol})$ in dry THF $(20 \mathrm{~mL})$ was then added dropwise. The resulting dark red solution was poured into $100 \mathrm{~mL}$ of ice water and $18 \mathrm{~mL}$ of acetic acid. The reaction was extracted with hexane $(3 \times 50 \mathrm{~mL})$ and the combined extracts were washed with $\mathrm{NaHCO}_{3}$, brine and dried with $\mathrm{MgSO}_{4}$. The residue $(6 \mathrm{~g}, 86 \%)$ of ethyl $(E)-3,5$-hexadienoate which was essentially pure was used for the next reaction without further purification. ${ }^{1} \mathrm{H}$ NMR (300 $\left.\mathrm{MHz}, \mathrm{CDCl}_{3}\right) \delta .32(1 \mathrm{H}, \mathrm{dt}, J=16.9,10.4), 6.12(1 \mathrm{H}, \mathrm{dd}, J=15.2,10.4), 5.77(1 \mathrm{H}, \mathrm{dt}, J$ $=15.2,7.2), 5.14(1 \mathrm{H}, \mathrm{d}, J=16.9), 5.04(1 \mathrm{H}, \mathrm{d}, J=10.1), 4.12(2 \mathrm{H}, \mathrm{q}, J=7.2), 3.09(2 \mathrm{H}$, d, $J=7.2), 1.23(3 \mathrm{H}, \mathrm{t}, J=7.2) ;{ }^{13} \mathrm{C} \mathrm{NMR}\left(75 \mathrm{MHz}, \mathrm{CDCl}_{3}\right) \delta 171.1,136.1,133.9$, $135.4,116.5,60.4,37.7,13.8$.

The above ester was dissolved in ether $(5.4 \mathrm{~g}, 38.6 \mathrm{mmol})$ and slowly added to a well-stirred suspension of lithium aluminum hydride $(2.0 \mathrm{~g}, 52.6 \mathrm{mmol})$ in ether $(40 \mathrm{~mL})$. The reaction was allowed to stir at room temperature overnight followed by quenching by sequential addition of water $(2 \mathrm{~mL}), 2 \mathrm{M} \mathrm{NaOH}(2 \mathrm{~mL})$ and water $(6 \mathrm{~mL})$. The mixture was filtered and the filtrate washed with brine and dried with $\mathrm{MgSO}_{4}$. The solvent was removed under vacuum and the residue purified by distillation to give 3,5-hexadien-1-ol as a clear oil (4.9 g, 92\%): bp 79-80 ${ }^{\circ} \mathrm{C}(20 \mathrm{~mm}) ;{ }^{1} \mathrm{H}$ NMR $\left(300 \mathrm{MHz}, \mathrm{CDCl}_{3}\right) \delta 6.65-$ $4.85(5 \mathrm{H}, \mathrm{m}), 3.63(2 \mathrm{H}, \mathrm{t}, J=6.1), 3.01(1 \mathrm{H}, \mathrm{bs}), 2.32(2 \mathrm{H}, \mathrm{q}, J=6.1) ;{ }^{13} \mathrm{C} \mathrm{NMR}(75$ $\left.\mathrm{MHz}, \mathrm{CDCl}_{3}\right) \delta(137.1,133.5,130.8,115.7,61.87,35.9$. 
tert-Butoxycarbonylimino-[(6-(2-hydroxyethyl)-3,6-dihydro-[1,2]oxazin-2-yl)]methyl-carbamic acid tert-butyl ester (Table 3, entry 6).

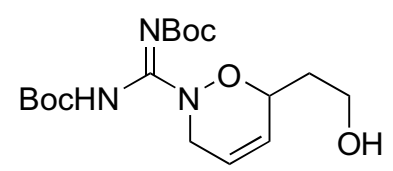

Obtained as a white solid in $74 \%$ yield: $\mathrm{Mp}=135-137{ }^{\circ} \mathrm{C}(\mathrm{dec}) ; \mathrm{R}_{f}=0.17$ (3:2 Hexanes: EtOAc); IR (KBr) v 3420 (broad), 2979, 1756, 1633, 1537, 1290, $1151 \mathrm{~cm}^{-1}$; ${ }^{1} \mathrm{H}$ NMR $\left(300 \mathrm{MHz}, \mathrm{CDCl}_{3}\right) \delta 9.91(1 \mathrm{H}, \mathrm{s}), 5.81(2 \mathrm{H}, \mathrm{m}), 4.86(1 \mathrm{H}, \mathrm{m}), 4.11(2 \mathrm{H}, \mathrm{q}, J=17.4,12.0$ $\mathrm{Hz}), 3.84(1 \mathrm{H}, \mathrm{m}), 3.76(1 \mathrm{H}, \mathrm{m}), 2.75(1 \mathrm{H}, \mathrm{bs}), 1.84(3 \mathrm{H}, \mathrm{m}), 1.49(18 \mathrm{H}, \mathrm{s}) ;{ }^{13} \mathrm{C} \mathrm{NMR}$ $\left(75 \mathrm{MHz}, \mathrm{CDCl}_{3}\right) \delta 152.9,127.7,121.9,77.9,59.7,46.04,42.76,35.03,28.15$ (1 quaternary carbon not observed); MS (EI) m/e (rel intensity) 372 (2), 242 (21), 214 (14), 171 (14), 129 (16), 84 (18), 67 (25), 57 (100); HRMS (EI) m/e (M ${ }^{+}$) calcd (for $\left.\mathrm{C}_{17} \mathrm{H}_{30} \mathrm{~N}_{3} \mathrm{O}_{6}\right)$ 372.2134, found 372.2141.

tert-Butoxycarbonylimino-(6-hydroxymethyl-3-methyl-3,6-dihydro-[1,2]oxazin-2yl)-methyl]-carbamic acid tert-butyl ester (Table 3, entry 7).

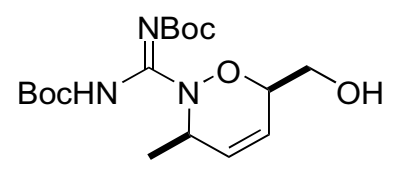

Obtained as a clear oil in 60\% yield: $\mathrm{R}_{f}=0.30$ (EtOAc:hexanes; 1:1); IR (Thin Film) v 3283, 2978, 2933, 1757, 1598, 1368, 1291, 1253, $1135 \mathrm{~cm}^{-1} ;{ }^{1} \mathrm{H}$ NMR (300 MHz, $\left.\mathrm{CDCl}_{3}\right) \delta 5.90(1 \mathrm{H}, \mathrm{ddd}, J=10.3,4.7,2.3 \mathrm{~Hz}), 5.66(1 \mathrm{H}, \mathrm{ddd}, J=10.3,1.5,1.5 \mathrm{~Hz})$, 4.87-4.85 (1H, m), $4.79(1 \mathrm{H}, \mathrm{m}), 3.76(1 \mathrm{H}, \mathrm{dd}, J=12.3,3.0 \mathrm{~Hz}), 3.67(1 \mathrm{H}, \mathrm{dd}, J=12.3$, $6.9 \mathrm{~Hz}), 1.49(18 \mathrm{H}, \mathrm{s}), 1.34(3 \mathrm{H}, \mathrm{d}, J=6.6 \mathrm{~Hz}) ;{ }^{13} \mathrm{C} \mathrm{NMR}\left(100 \mathrm{MHz}, \mathrm{CDCl}_{3}\right) \delta 150.91$, $130.04,123.09,80.66,63.09,51.30,28.63,28.46,17.77$ (1 quaternary carbon not 
observed); MS (EI) m/e (rel intensity) 372 (7), 260 (15), 242 (28), 184 (37), 149 (34), 111 (44), 98 (66), 81 (98), 57 (100); HRMS (EI) m/e calcd $\left(\mathrm{M}^{+}\right)$372.2134, found 372.2130.

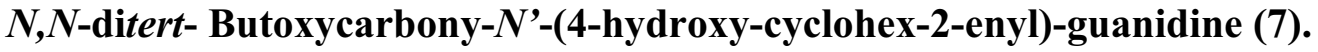<smiles>CC(C)(C)OC(=O)NC(=N[S+](=O)[O-])NC1C=CC(O)CC1</smiles>

A solution of [tert-butoxycarbonylimino-(2-oxa-aza-bicyclo[2.2.2]oct-5-en-3-yl)methyl]-carbamic acid tert-butyl ester $(0.200 \mathrm{~g}, 0.56 \mathrm{mmol})$ and $\mathrm{Na}_{2} \mathrm{HPO}_{4}(0.37 \mathrm{~g}, 2.60$ mmol, ) in dry ethanol was cooled to $0^{\circ} \mathrm{C}$ in an ice water bath. To this was added $\mathrm{Na}(\mathrm{Hg})$ amalgam $(0.7 \mathrm{~g})$ in small portions and the solution allowed to stir for $10 \mathrm{~h}$. The reaction was next filtered through a pad of celite and concentrated to a brown solid. The product was purified by silica gel column chromatography to give the desired compound as a white solid (0.37 g, 95\%). Mp = 205-208 C(dec); $\mathrm{R}_{f}=0.25$ (3:2 Hexanes: EtOAc); IR $(\mathrm{KBr}) \vee 3323$ (broad), 2980, 1721, 1612, 1415, 1314, 1252, $1157 \mathrm{~cm}^{-1}$; ${ }^{1} \mathrm{H}$ NMR (300 $\left.\mathrm{MHz}, \mathrm{CDCl}_{3}\right) \delta 5.90(1 \mathrm{H}, \mathrm{m}), 5.74(1 \mathrm{H}, \mathrm{m}), 4.66(1 \mathrm{H}, \mathrm{bs}), 4.17(1 \mathrm{H}, \mathrm{bs}), 2.32(1 \mathrm{H}, \mathrm{bs})$, 1.90-1.70 (4H, m), 1.49 (9H, s), $\left.1.49(9 \mathrm{H}, \mathrm{s}) ;{ }^{13} \mathrm{C} \mathrm{NMR} \mathrm{(75} \mathrm{MHz,} \mathrm{CDCl}_{3}\right) \delta 161.4,153.3$, 150.9, 130.6, 128.1, 81.2, 77.4, 62.5, 43.7, 27.3, 26.6, 26.3, 23.4; HRMS (EI) m/e (M+) calcd (for $\mathrm{C}_{17} \mathrm{H}_{29} \mathrm{~N}_{3} \mathrm{O}_{5}$ ) 355.2107, found 355.2109. 
[tert-Butoxycarbonylimino-(2-oxa-3-aza-bicyclo[2.2.2]oct-3-yl)-methyl]-carbamic acid tert-butyl ester (8).

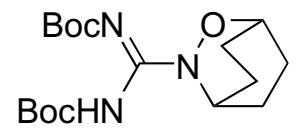

A ballon of $\mathrm{H}_{2}$ was passed over a suspension of [tert-Butoxycarbonylimino-(2-oxa-azabicyclo[2.2.2] oct-5-en-3-yl)-methyl]-carbamic acid tert-butyl ester (100 mg, $0.28 \mathrm{mmol})$ and $\mathrm{Pd}(\mathrm{OH})_{2}(5 \% \mathrm{mmol}, 10 \mathrm{mg})$ in $3 \mathrm{ml}$ of $\mathrm{MeOH}$ for 12 hours. The reaction was filtered through a pad of celite and concentrated. The resulting white solid was purified by silica gel chromatography to give the desired product as a white solid $(51.3 \mathrm{mg}, 70 \%) . \mathrm{mp}=$ $205{ }^{\circ} \mathrm{C} ; \mathrm{R}_{f}=0.20$ (EtOAc:hexanes; 3:2); IR (Thin Film) v 3289, 2975, 1759, 1682, 1600, 1504, 1153, $951 \mathrm{~cm}^{-1} ;{ }^{1} \mathrm{H}$ NMR (300 MHz, $\left.\mathrm{CDCl}_{3}\right) \delta 4.51(1 \mathrm{H}, \mathrm{bs}), 4.31(1 \mathrm{H}, \mathrm{bs}), 2.29-$ $2.12(4 \mathrm{H}, \mathrm{m}), 1.77-1.67(4 \mathrm{H}, \mathrm{m}), 1.47(18 \mathrm{H}, \mathrm{s}) ;{ }^{13} \mathrm{C} \mathrm{NMR}\left(75 \mathrm{MHz}, \mathrm{CDCl}_{3}\right) \delta 150.26$, 73.76, 48.28, 28.15, 24.93, 23.44 (1 quaternary carbon not observed); MS (EI) m/e (rel intensity) 355 (7), 225 (29), 208 (23), 181 (15), 113 (75), 81 (55), 70 (35), 57 (100); HRMS (EI) m/e calcd $\left(\mathrm{M}^{+}\right)$355.2107, found 355.2105.

\section{$N, N$-di-tert- Butoxycarbony- $N$ '-(4-hydroxy-cyclohexyl)-guanidine (9).}

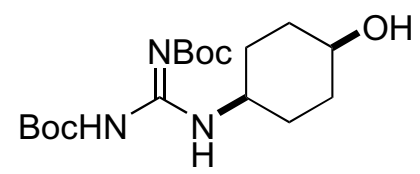

A ballon of $\mathrm{H}_{2}$ was passed over a suspension of [tert-butoxycarbonylimino-(2-oxa-3-azabicyclo[2.2.2] oct-3-yl)-methyl]-carbamic acid tert-butyl ester $(0.20 \mathrm{mmol}, 74 \mathrm{mg})$ and Raney nickel $(5 \%, 7.4 \mathrm{mg})$ in $\mathrm{MeOH}(5 \mathrm{~mL})$ for 5 hours. The reaction was filtered through a pad of celite and the concentrated to a clear oil. The resulting oil was purified by silica gel chromatography with 3:2 hexanes:EtOAc to give the desired product as a white solid (67.7 mg, 91\%). $\mathrm{mp}=183{ }^{\circ} \mathrm{C}$; $\mathrm{R}_{f}=0.25$ (EtOAc:hexanes; 2:3); IR (Thin 
Film) v 3434(broad), 2979, 2934, 1719, 1639, 1416, 1368, 1131, $1056 \mathrm{~cm}^{-1} ;{ }^{1} \mathrm{H}$ NMR $\left(300 \mathrm{MHz}, \mathrm{CDCl}_{3}\right.$, rotomers) $\delta 11.51(1 \mathrm{H}, \mathrm{s}), 8.49(1 \mathrm{H}, \mathrm{d}, J=7.6 \mathrm{~Hz}), 4.12(1 \mathrm{H}, \mathrm{m}), 3.87$ $(1 \mathrm{H}, \mathrm{bs}), 1.72(8 \mathrm{H}, \mathrm{m}), 1.50(9 \mathrm{H}, \mathrm{s}), 1.49(9 \mathrm{H}, \mathrm{s}) ;{ }^{13} \mathrm{C} \mathrm{NMR}\left(75 \mathrm{MHz}, \mathrm{CDCl}_{3}\right) \delta 163.84$, $155.38,153.31,83.01,79.13,66.87,46.74,31.11,28.34,28.11,27.40$; MS (EI) m/e (rel intensity) 357 (9), 301 (15), 245 (75), 228 (26), 200 (21), 148 (66), 81 (22), 57 (100); HRMS (EI) m/e calcd ( $\left.\mathbf{M}^{+}\right)$357.2264, found 357.2265.

\section{$N$-(4-hydroxy-cyclohexyl)-guanidine hydrochloride (10).}

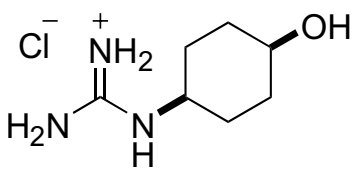

$9(0.112 \mathrm{mmol}, 0.040 \mathrm{~g})$ was taken up in $\mathrm{MeOH}(3 \mathrm{~mL})$ and to this was added $0.22 \mathrm{~mL}$ of $2 \mathrm{M} \mathrm{HCl}$ and the reaction allowed to stir for 4 hours at room temperature. The water was then removed under vacuum as an azeotrope with toluene and the resulting white solid was purified through a small plug of silica gel (9:1 DCM: $\mathrm{MeOH})$ to give 9 as a white solid (0.0192 g, 89\%): $\mathrm{mp}=115{ }^{\circ} \mathrm{C} ; \mathrm{R}_{f}=0.32$ (DCM:MeOH; 9:1); IR (KBr Pellet) v 3200, 2932, 1720, 1326, 1100, $936 \mathrm{~cm}^{-1} ;{ }^{1} \mathrm{H}$ NMR $\left(300 \mathrm{MHz}, \mathrm{CDCl}_{3}\right) \delta 3.89(1 \mathrm{H}, \mathrm{m})$, $3.34(1 \mathrm{H}, \mathrm{m}), 1.73(8 \mathrm{H}, \mathrm{m}) ;{ }^{13} \mathrm{C} \mathrm{NMR}\left(100 \mathrm{MHz}, \mathrm{CDCl}_{3}\right) \delta 156.31,64.94,48.98,30.27$, 26.65; MS (EI) m/e (rel intensity) 193 (5), 149 (10), 120 (11), 105 (100), 91 (50), 77 (21), 65 (11); HRMS (EI) m/e calcd ( $\left.\mathrm{M}^{+}\right)$193.6736, found 193.6741. 